\title{
Кинетика и механизм адсорбции сульфат-ионов на активированном угле КМ-2
}

\author{
(C) 2021 Свешникова Д.А. ${ }^{1,2}$, Сулейманов С.И., ${ }^{2,3}$, Хамизов Р.Х. ${ }^{4}$ \\ ${ }^{1}$ Институт проблем геотермии и возобновляемой энергетики \\ Филиал Объединенного Института высоких температур РАН, Махачкала \\ ${ }^{2}$ Аналитический центр коллективного пользования ДФИЦ РАН, Махачкала \\ ${ }^{3}$ Дагестанский государственный университет, Махачкала \\ ${ }^{4}$ Институт аналитической химии и геохимии им. В.И. Вернадского (ГЕОХИ) РАН, Москва \\ Поступила в редакцию 15.06.2021 г.
}

DOI: $10.17308 /$ sorpchrom.2021.21/3635

Статья посвящена изучению кинетики адсорбции и электросорбции (адсорбции под катодным потенциалом) ионов $\mathrm{SO}_{4}{ }^{2-}$ на активированном угле КМ-2. Исследования проведены в статическом режиме в ограниченном объеме раствора. Экспериментальные данные по кинетике адсорбции $\mathrm{SO}_{4}{ }^{2-}$ на неполяризованном и поляризованном угле были обработаны с использованием кинетических диаграмм для смешанной диффузии и линейной изотермы. Сопоставление экспериментальных данных с теоретическими зависимостями показало, что адсорбция сульфата на неполяризованном угле лучше описывается уравнением смешаннодиффузионной кинетики близкой к внешней диффузии, чем электросорбция этих ионов.

Обнаружено, что катодная поляризация увеличивает величину сорбции данных ионов, кроме того, при электросорбции в условиях катодной поляризации существенная часть ионов $\mathrm{SO}_{4}{ }^{2-}$ сорбируется необратимо, избыточная часть адсорбтива не десорбируется при изменении поляризации. Исследования показали, что процессы адсорбции ионов $\mathrm{SO}_{4}{ }^{2-}$ на неполяризованном и катодно-поляризованном угле КМ-2 могут быть удовлетворительно описаны моделями формальной кинетики, допускающей возможность химического лимитирования скорости таких процессов. Исходя из факта необратимости адсорбции исследуемых ионов, сделан вывод о влиянии химического механизма торможения на кинетику процесса электросорбции сульфата на АУ КМ-2 в условиях катодной поляризации.

Полуэмпирическим методом РМ3 с помощью программы Gaussian 09W проведено квантовохимическое изучение процесса адсорбции сульфат-иона на поверхности активированного угля, моделируемого кислородсодержащим углеродным кластером $\mathrm{C}_{97} \mathrm{H}_{22} \mathrm{O}_{6}$. Показано, что ионы $\mathrm{SO}_{4}{ }^{2-}$ могут давать прочные соединения с углеродным кластером.

Ключевые слова: адсорбция, элетросорбция, сульфат-ион, смешаннодиффузионная кинетика, псевдо-первый, псевдо-второй порядок, квантово-химические расчеты.

\section{Введение}

Сульфат-ионы являются наиболее распространенными загрязнителями, присутствующими, как в природных, так и сточных водах различных производств. Основным природным источником ионов $\mathrm{SO}_{4}{ }^{2-}$ является выветривание и растворение серосодержащих минералов. Загрязнение природных вод этими ионами может быть обусловлено и человеческой де- ятельностью, в частности, сбросом в открытые водоемы недостаточно очищенных сточных вод химической и текстильной промышленности дубильных, кожевенных производств.

Несмотря на то, что ионы $\mathrm{SO}_{4}{ }^{2-}$ менее токсичны, чем ионы тяжелых металлов, содержание их в питьевых водах строго регламентировано (по требованиям ВО3, EC и USEPA это количество не должно превышать 250 мг/дм $\left.{ }^{3}\right)$. Содержание 
сульфатов в бытовых сточных водах регламентируется возможностями региональных очистных систем, но, как правило, оно не должно превышать $500 \mathrm{мг} /$ дм $^{3}$.

Для удаления сульфат-ионов из водных растворов с различной степенью эффективности используют такие методы, как осаждение [1], кристаллизация [2], ионный обмен [3], электродиализ [4], нанофильтрация [5], обратный осмос [6], адсорбция. Все эти методы имеют свои недостатки и ограничения. Наиболее предпочтительным является адсорбционный метод. В качестве сорбентов используются гидроксиды железа [7], алюминия [8], различные глинистые минералы [9], активированные угли [10-13], являющиеся весьма эффективными сорбентами, благодаря высокоразвитой поверхности, пористости и возможности выбора материалов с различными поверхностными функциональными группами.

Исследования показали [13], что лучшими сорбентами для удаления из водных растворов анионов, и в частности ионов $\mathrm{SO}_{4}{ }^{2-}$, являются активированные угли, на поверхности которых присутствуют функциональные группы основного характера. В работе [13] отмечается также, что в противоположность карбоксильным и лактонным поверхностным группам, которые не участвуют в процессе адсорбции анионов на АУ, фенольные группы такое участие принимают, но механизм адсорбции анионов на этих группах не был раскрыт.

Нами ранее $[14,15]$ была обнаружена эффективная адсорбция сульфат-ионов на активированном угле КМ-2, на поверхности которого имеются только функциональные группы кислотного характера. Показана также возможность увеличения сорбционной емкости данного угля по этим анионам путем катодной поляризации сорбента, приводящей к наработке на его поверхности функциональных групп основного характера [14].
Целью настоящей работы является установление кинетических закономерностей и механизма адсорбции ионов $\mathrm{SO}_{4}{ }^{2-}$ на неполяризованном и катоднополяризованном угле КМ-2.

\section{Экспериментальная часть}

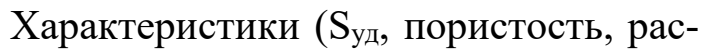
пределение пор по размерам, элементный состав, содержание поверхностных функциональных групп) используемого в данной работе активированного угля КМ-2 приведены в работах [15-17].

Исследование кинетики процесса адсорбции и электросорбции ионов $\mathrm{SO}_{4}^{2-}$ проводили в статическом режиме. Адсорбцию осуществляли в конической колбе объемом $250 \mathrm{~cm}^{3}$, в которую помещали активированный уголь и добавляли раствор, содержащий ионы $\mathrm{SO}_{4}{ }^{2-}$ (соотношение «сорбент: объем раствора» составляло 1:40), смесь перемешивали с помощью магнитной мешалки. Процесс электросорбции (сорбции под катодным потенциалом) ионов $\mathrm{SO}_{4}{ }^{2-}$ осуществляли в электрохимической ячейке с разделенными анодным и катодным пространствами. Токоподводом служил графитовый стержень, в качестве анода использовали графит, плотность тока составляла $30 \mathrm{~mA} /$ г. Подробное описание электросорбционных экспериментов дано в [18]. В обоих случаях концентрация исходного раствора сульфата натрия, который готовили из соли $\mathrm{Na}_{2} \mathrm{SO}_{4}$ («Хч») в фоновом растворе $\mathrm{NaCl}\left(50 \Gamma^{2} / \mathrm{sm}^{3}\right)$, составляла 1700 мг/дм ${ }^{3}$, необходимое значение $\mathrm{pH}$ (1.50-1.75) достигали добавлением раствора $\mathrm{HCl}$. Адсорбцию ионов $\mathrm{SO}_{4}{ }^{2-}$ оценивали по убыли их в растворе. Концентрацию сульфат-ионов до и после адсорбции определяли весовым методом [19].

\section{Обсуждение результатов}

Из рис.1 представлены кинетические кривые адсорбции и электросорбции ионов $\mathrm{SO}_{4}{ }^{2-}$ на активированном угле КМ2 , из которых видно, что катодная поляризация увеличивает величину сорбции 


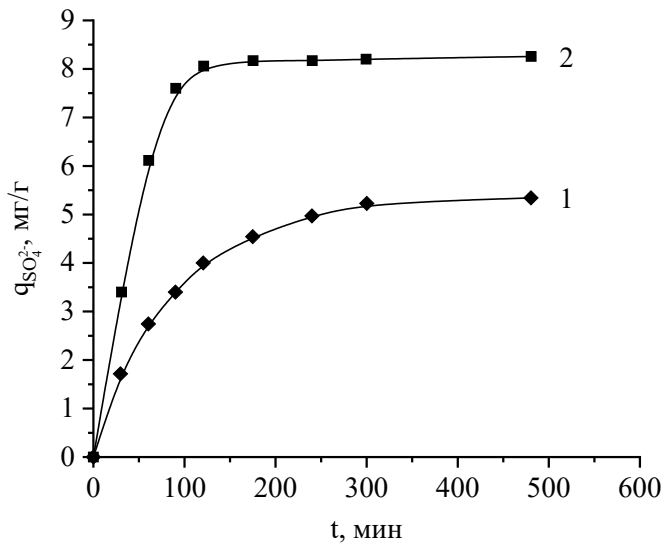

Рис.1. Кинетические кривые адсорбции $\mathrm{SO}_{4}{ }^{2-}$ на неполяризованном (1) и поляризованном (2) угле КМ-2

Fig. 1. Kinetic curves of the $\mathrm{SO}_{4}{ }^{2-}$ adsorption on nonpolarised (1) and polarised (2) KM-2 carbon

данных ионов и сокращает время достижения равновесного значения сорбции.

Изотермы адсорбции ионов $\mathrm{SO}_{4}{ }^{2-}$, полученные при используемых способах сорбции приведены на рис. 2 , из которого видно, что, если изотерма на неполяризованном сорбенте практически прямолинейна, изотерма электросорбции имеет слабовыпуклую форму.

Для описания гетерогенных процессов адсорбции могут быть использованы стандартные подходы, основанные на диффузионных представлениях или на уравнениях формальной кинетики, в частности, моделях псевдо-первого и псевдо-второго порядков, чаще всего применяемых для процессов, лимитируемых химическим механизмом. В последнее время благодаря работам [20-23], к которым следует добавить и наши работы $[24,25]$, стало ясно, что уравнения формальной кинетики для адсорбции могут описывать и диффузионные процессы. Следует также иметь ввиду, что формализации для химических процессов первого порядка и внешнедиффузионных процессов (также как и асимптотических приближений для внутренней диффузии при больших временах) могут полностью совпадать [21]. Это требует дополнительных исследований для выявления кинетических механизмов.

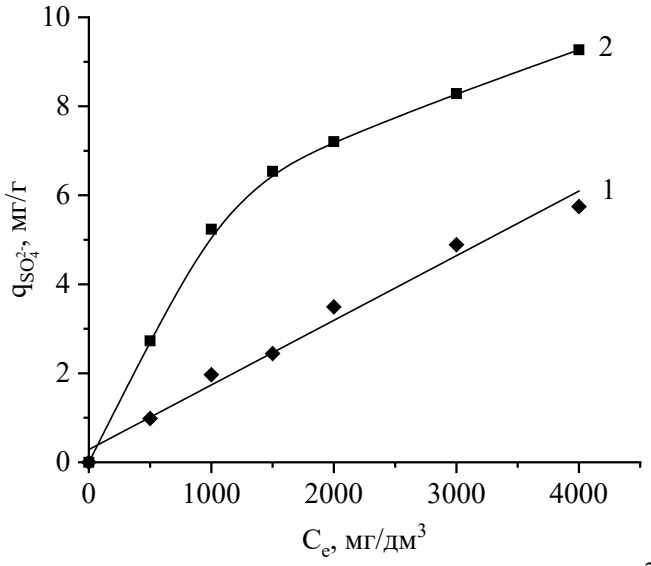

Рис. 2. Изотерма адсорбции ионов $\mathrm{SO}_{4}{ }^{2-}$ при температуре $25^{\circ} \mathrm{C}$ на неполяризованном (1) и поляризованном (2) угле КМ-2

Fig. 2. The isotherm of the $\mathrm{SO}_{4}{ }^{2-}$ ions at a temperature of $25^{\circ} \mathrm{C}$ on nonpolarised (1) and polarised (2) KM-2 carbon

Наиболее общим подходом при использовании диффузионной кинетики является предположение о смешанно-диффузионном механизме и сопоставление экспериментальных данных с теоретическими зависимостями при варьировании значений диффузионного критерия [26]:

$$
H=\frac{\beta \cdot r^{2}}{\bar{D} \Gamma}
$$

где $\beta$ - коэффициент массопереноса $\left(\mathrm{c}^{-1}\right)$; $\bar{D}$ - коэффициент внутренней диффузии $\left(\mathrm{cm}^{2} / \mathrm{c}\right) ; \Gamma-$ равновесный коэффициент распределения при линейной изотерме и $\mathrm{r}$ - средний радиус частиц адсорбента.

Для того чтобы не проводить каждый раз расчеты с использованием весьма сложных формул в виде сходящихся бесконечных рядов, наиболее удобным представляется использование существующих библиотек решений в графическом виде в большом диапазоне значений диффузионного критерия от $\mathrm{H}>>1$, когда кинетика процесса лимитируется внутренней диффузией, до $\mathrm{H} \rightarrow 0$ для процессов контролируемых внешней диффузией [27]

На рис 3 (а и б) и в табл. 1 приведены результаты обработки экспериментальных данных по адсорбции сульфат-ионов на неполяризованном и поляризованном угле КМ-2 с использованием расчетных кинетических диаграмм для смешанной 

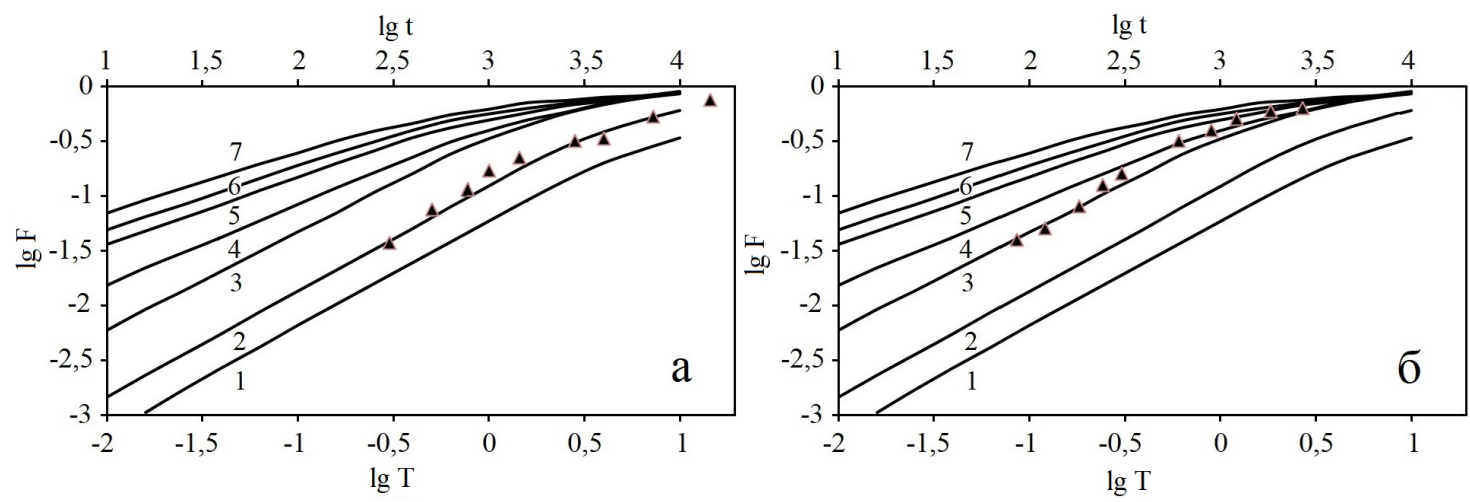

Рис. 3. Теоретические зависимости $\mathrm{F}(\mathrm{H}, \mathrm{T})$ при смешанно-диффузионной кинетике при значениях $\mathrm{H}: 1-1 ; 2-2 ; 3-8 ; 4-20 ; 5-63 ; 6-100 ; 7-\infty$ [26] и экспериментальные данные: а - адсорбция $\mathrm{SO}_{4}{ }^{2-}$ на неполяризованном угле КМ-2; б - адсорбция $\mathrm{SO}_{4}{ }^{2-}$ на катоднополяризованном угле КМ-2

Fig. 3. Theoretical dependences $\mathrm{F}(\mathrm{H}, \mathrm{T})$ for mixed-diffusion kinetics for different values of $\mathrm{H}: 1-1 ; 2-2 ; 3-8 ; 4-20 ; 5-63 ; 6-100 ; 7-\infty[26]$ and the experimental data: $\mathrm{a}-$ adsorption of $\mathrm{SO}_{4}{ }^{2-}$ on nonpolarised $\mathrm{KM}-2$ carbon; $\mathrm{b}$ - adsorption of $\mathrm{SO}_{4}{ }^{2-}$ on cathode-polarised KM-2 carbon

Таблица 1. Диффузионные параметры адсорбции сульфат-ионов на неполяризованном и поляризованном угле КМ-2

Table 1. Diffusion parameters of the adsorption of sulphate ions on nonpolarised and polarised KM-2 carbon

\begin{tabular}{|c|c|c|c|}
\hline Способ адсорбции & $\beta, \mathrm{c}^{-1}$ & $\bar{D}, \mathrm{~cm}^{2} / \mathrm{c}$ & $H$ \\
\hline Без поляризации & $1.91 \cdot 10^{-3}$ & $1.09 \cdot 10^{-6}$ & 2 \\
\hline Катодная поляризация & $2.60 \cdot 10^{-3}-8.00 \cdot 10^{-2}$ & $2.99 \cdot 10^{-7}-3.33 \cdot 10^{-7}$ & $8-20$ \\
\hline
\end{tabular}

диффузии и линейной изотермы, приведенных в [26].

На этих диаграммах; $T=\beta r / \Gamma-$ т.н. «безразмерное» время, $F=q_{t} / q_{e}-$ степень завершения кинетического процесса, $\mathrm{q}_{\mathrm{t}}$ и $\mathrm{q}_{\mathrm{e}}$ - текущая и равновесная концентрации целевого компонента.

Результаты, приведенные на этих рисунках, показывают, что с некоторым приближением процесс адсорбции сульфата на неполяризованном угле описывается моделью смешанной диффузии близкой к внешнедиффузионному процессу. Чуть более сложным является описание кинетики адсорбции на катоднополяризованном угле, где мы не наблюдаем точного соответствия модели, так как по ходу процесса имеет места небольшое смещение от кривой, соответствующей $\mathrm{H}=8$, до зависимости для $\mathrm{H}=20$, что может быть связано с тем, что эксперименталь- ная область изучения кинетики находится в слабовыпуклой части изотермы электросорбции.

Экспериментальные данные были обработаны также по уравнениям псевдопервого (1), псевдо-второго (2) порядков $[28,29]$. Результаты приведены на рис.4 и в табл. 2.

$$
\begin{gathered}
\ln \left(q_{e}-q_{t}\right)=\ln q_{e}-k_{1} t \\
\frac{t}{q_{t}}=\frac{1}{k_{2} q_{e}^{2}}+\frac{1}{q_{e}} t
\end{gathered}
$$

Сравнение результатов использования моделей псевдо-первого и псевдо-второго порядков (табл.2) для описания кинетики сорбции исследуемых ионов показывает, что адсорбция $\mathrm{SO}_{4}{ }^{2-}$ на неполяризованном АУ удовлетворительно описывается обеими моделями, а в случае адсорбции этих ионов на поляризованном угле уравнение псевдо-второго порядка позволяет описать экспериментальные 

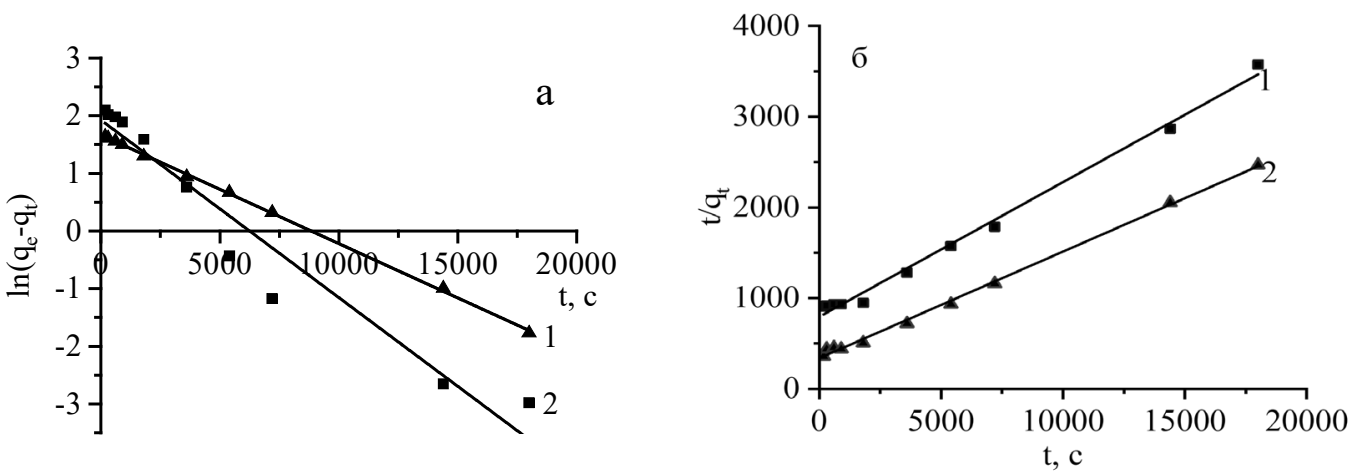

Рис. 4. Модели псевдо-первого (а) и псевдо-второго (б) порядков адсорбции $\mathrm{SO}_{4}{ }^{2-}$ : 1 - на неполяризованном угле КМ-2; 2 - на катоднополяризованном угле КМ-2

Fig. 4. Models of the $\mathrm{SO}_{4}{ }^{2-}$ adsorption of pseudo-first (a) and pseudo-second (b) orders: 1 - on nonpolarised KM-2 carbon; 2 - on cathode-polarised KM-2 carbon

Таблица 2. Экспериментальные значения $\mathrm{q}_{\mathrm{e}}$ и коэффициенты уравнений псевдо-первого, псевдо-второго порядков ионов $\mathrm{SO}_{4}{ }^{2-}$ на неполяризованном и катоднополяризованном АУ KM-2

Table 2. Experimental values of $\mathrm{q}_{\mathrm{e}}$ and the coefficients of the equations of the $\mathrm{SO}_{4}{ }^{2-}$ ions of pseudofirst and pseudo-second orders on nonpolarised and cathode-polarised KM-2 activated carbon

\begin{tabular}{|c|c|c|c|c|c|c|c|}
\hline \multirow{2}{*}{$\begin{array}{c}\text { Способ } \\
\text { адсорб- } \\
\text { ции }\end{array}$} & \multicolumn{3}{|c|}{ Псевдо-первый порядок } & \multicolumn{3}{|c|}{ Псевдо-второй порядок } & \multirow[b]{2}{*}{$\begin{array}{c}\mathrm{q}_{\mathrm{e}} \text { эксп } \\
\text { МГ/Г }\end{array}$} \\
\hline & $\begin{array}{c}\mathrm{k}_{1} \cdot 10^{4} \\
\mathrm{c}^{-1}\end{array}$ & $\mathrm{q}_{\mathrm{e}}, \mathrm{M \Gamma} / \Gamma$ & $\mathrm{R}^{2}$ & $\begin{array}{c}\mathrm{k}_{2} \cdot 10^{5} \\
\Gamma \cdot \mathrm{M} \Gamma^{-1} \cdot \mathrm{c}^{-1}\end{array}$ & $\mathrm{q}_{\mathrm{e}}, \mathrm{M \Gamma} / \mathrm{\Gamma}$ & $\mathrm{R}^{2}$ & \\
\hline $\begin{array}{c}\text { Без по- } \\
\text { ляриза- } \\
\text { ции }\end{array}$ & 2.04 & 5.28 & 0.999 & 2.60 & 6.95 & 0.995 & 5.27 \\
\hline $\begin{array}{c}\text { Катод- } \\
\text { ная по- } \\
\text { ляриза- } \\
\text { ция }\end{array}$ & 3.40 & 6.84 & 0.945 & 3.68 & 8.58 & 0.998 & 8.20 \\
\hline
\end{tabular}

данные с более высокими коэффициентами корреляции $\mathrm{R}^{2}$, и значения $q_{e}$, рассчитанные по этому уравнению дают лучшее совпадение с экспериментальными. Само по себе это не является доказательством химического механизма кинетики адсорбции [24-26]. Однако, в пользу этого, или, как минимум, в пользу соизмеримости влияния химического и диффузионного торможения, говорят не только приведенные выше результаты обработки экспериментальных данных по диффузионной модели. Дополнительным фактором, подтверждающим роль химической кинетики, является то, что существенная часть сульфат-ионов, адсорбируемых при катодной поляризации и определяющих повышенную емкость поляризованного АУ, связывается необратимо и не десорбируется в условиях анодной поляризации.
Представляет интерес выявление роли поверхностных функциональных групп в адсорбции сульфат-иона на исследуемых углях. Методом Боэма [30] было установлено, что на угле КМ-2 с адсорбированными сульфат-ионами количество карбоксильных групп сокращается с 0.453 до 0.06 мг-экв/г. Суммарное количество лактонных и фенольных групп остается практически неизменным. В работе [31] отмечается, что на активированных углях извлечение ионов $\mathrm{SO}_{4}{ }^{2-}$ из водных растворов базируется на электростатическом взаимодействии адсорбат-адсорбент, которое зависит от поверхностной функциональности.

Для выяснения механизма сорбции сульфат-ионов $\mathrm{SO}_{4}{ }^{2-}$ на активированном угле полуэмпирическим методом РМ3 с помощью программы Gaussian 09W проведен квантово-химический расчет 


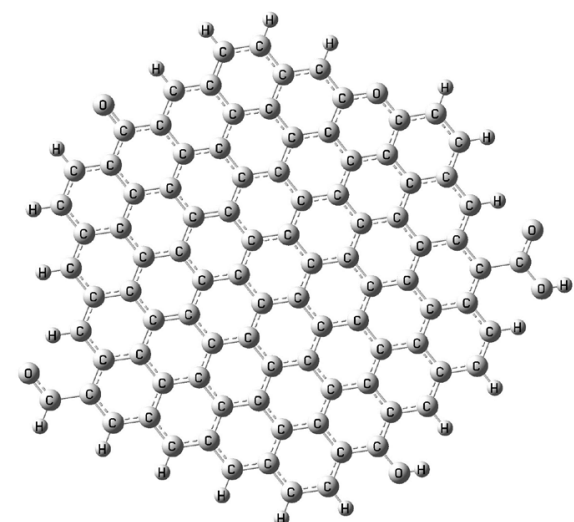

a

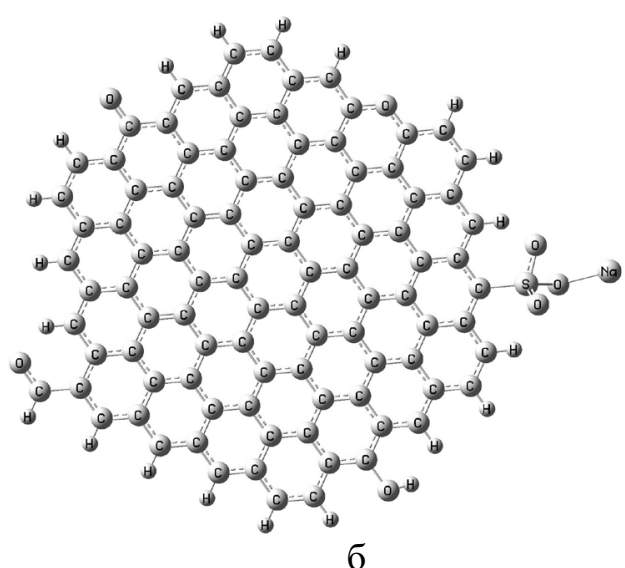

б

Рис. 5. Кислородсодержащий углеродный кластер (а) и его предполагаемый сульфированный продукт хемосорбции (б)

Fig. 5. Oxygen-containing carbon cluster

Таблица 3. Рассчитанные энергетические параметры реактантов по данным метода РМ3 Table 3. Full energy, the heat of the formation, and dipole moment of reactants according to the PM3 data

\begin{tabular}{|c|c|c|c|}
\hline Реактант & $\begin{array}{c}\text { Внутренняя энергия } \\
(\Delta \mathrm{E}), \text { кДж/моль }\end{array}$ & $\begin{array}{c}\text { Энтальпия }(\Delta \mathrm{H}), \\
\text { кДж/моль }\end{array}$ & $\begin{array}{c}\text { Энергия Гиббса }(\Delta \mathrm{G}), \\
\text { кДж/моль }\end{array}$ \\
\hline $\mathrm{C}_{97} \mathrm{H}_{22} \mathrm{O}_{6}$ & 3088.3 & 3094.3 & 2718.9 \\
\hline $\mathrm{C}_{96} \mathrm{H}_{21} \mathrm{O}_{4} \mathrm{SO}_{3} \mathrm{Na}$ & 2787.3 & 2791.8 & 2400.6 \\
\hline $\mathrm{CO}_{2}$ & -331.4 & -328.7 & -393.4 \\
\hline $\mathrm{H}_{2} \mathrm{O}$ & -170.4 & -167.9 & -224.0 \\
\hline $\mathrm{H}_{3} \mathrm{O}^{+}$ & 469.0 & 471.8 & 416.4 \\
\hline$\left[\mathrm{Na}_{2}\left(\mathrm{H}_{2} \mathrm{O}\right)\right]^{+}$ & -44.0 & -41.5 & -119.1 \\
\hline $\mathrm{SO}_{4}{ }^{2-}$ & -1464.2 & -1461.7 & -1544.8 \\
\hline
\end{tabular}

Таблица 4.Энергетические характеристики соединения иона $\mathrm{SO}_{4}{ }^{2-}$ с углеродным кластером, рассчитанные методом РМ3

Table 4. Energetic properties of the $\mathrm{SO}_{4}{ }^{2-}$ ion compound with the surface of a carbon cluster calculated by the PM3 method

\begin{tabular}{|c|c|c|c|}
\hline Система & $\begin{array}{c}\text { Энергия взаимо- } \\
\text { действия } \\
\Delta E_{r}, \text { кДж/моль }\end{array}$ & $\begin{array}{c}\text { Энтальпия } \\
\Delta H, \text { кДж/моль }\end{array}$ & $\begin{array}{c}\text { Энергия Гиббса } \\
\Delta \mathrm{G}, \text { кДж/моль }\end{array}$ \\
\hline $\mathrm{C}_{96} \mathrm{H}_{21} \mathrm{O}_{4} \mathrm{SO}_{3} \mathrm{Na}$ & -104.4 & -103.5 & -136.2 \\
\hline
\end{tabular}

энергетических параметров процесса хемосорбции этих ионов на кластерных молекулах активированного угля, моделируемого кислородсодержащим углеродным кластером $\mathrm{C}_{97} \mathrm{H}_{22} \mathrm{O}_{6}$ (рис. 5a). Предварительная геометрическая оптимизация соединений проводилась методом РМ3 с минимальной спиновой мультиплетностью и с учетом влияния растворителя $\left(\mathrm{H}_{2} \mathrm{O}\right)$ на уровне модельной теории СРСМ [32-34].

Рассчитанные методом РM3 энергетические параметры участвующих в реакции хемосорбции соединений (реактантов) приведены в табл. 3 и 4.

В области значений $1.5<\mathrm{pH}<1.75$ адсорбцию сульфата на выделенном кластере КМ-2 можно представить в виде взаимосвязанного двухстадийного процесса, включающего потерю карбоксильной группы и последующее присоединение к остаточному кластеру сульфогруппы (рис. 5б). Так как процесс адсорбции проводился из раствора с большим избытком ионов $\mathrm{Na}^{+}$ (экспериментально $\mathrm{Na}$ был обнаружен на поверхности угля после адсорбции $\mathrm{SO}_{4}{ }^{2-}$ ), 
расчеты проводили с учетом обмена $\mathrm{Na}^{+}$ на $\mathrm{H}^{+}$. Для оценочных расчетов результирующую реакцию можно представить следующим образом:

$$
\begin{gathered}
\mathrm{C}_{97} \mathrm{H}_{22} \mathrm{O}_{6}+\mathrm{SO}_{4}{ }^{2-}+\mathrm{H}_{3} \mathrm{O}^{+}+\left[\mathrm{Na}\left(\mathrm{H}_{2} \mathrm{O}\right)\right]^{+} \\
=\mathrm{C}_{96} \mathrm{H}_{21} \mathrm{O}_{4} \mathrm{SO}_{3} \mathrm{Na}+\mathrm{CO}_{2}+3 \mathrm{H}_{2} \mathrm{O}
\end{gathered}
$$

В соответствии с этим, и исходя из энергетических балансов, можно оценить энергию взаимодействия, энтальпию и энергию Гиббса этой реакции.

Даже с учетом того, что проведенные расчеты носят весьма приближенный характер, оценочные данные позволяют предполагать, что ион $\mathrm{SO}_{4}{ }^{2-}$ образует с углем КМ-2 весьма прочные связи.

\section{Заключение}

Исследования показали, что процессы адсорбции ионов $\mathrm{SO}_{4}{ }^{2-}$ на неполяризованном и катодно-поляризованном угле КМ2 могут быть удовлетворительно описаны моделями как диффузионной, так и формальной кинетики, допускающей возможность химического лимитирования скорости таких процессов.

\section{Список литературы}

1. Benatti C.T., Tavares C.R.G, Lenzi E. // J. Environ. Manag. 2009. Vol. 90. pp. 504-511

2. Tait S., Clarkeb W.P., Keller J., Batstonea D.J. // Water Research. 2009. Vol. 43. pp. $762-$ 772.

3. Basha C.A., Selvi S.J., Ramasamy E., Chellammal S. // Chem. Eng.J. 2008. Vol. 141. pp. 89-98.

4. Lee H.J., Oh S.J., Moon S.H. // Wat.Res. 2003. Vol. 37. pp.1091-1099.

5. Galliana-Aleixandre M.V., Iborra-Clar A., Bes-Pifi A., Mendoza-Roca J.A. et al. // Desalination. 2005. Vol. 179. pp. 307-313.

6. Bodalo A., Gomez J.L. Gomez E., Leon G. et al. // Desalination. 2004. Vol. 162. pp. 55-60.

7. Boukhalfa C. // Desalination. 2010. Vol. 250. pp. 428-432.

8. Kolics A., Polkinghorne J.C., Wieckowski A. // Electrochimica Acta. 1998. Vol. 43. pp. 2605-2618.

9. Ljng R., Liu Y., Wang X., Huang J. // J. Chem. Eng. Data. 2011. Vol. 56. pp. 3890-3896.

10. Rahmati, Yeganeh G., Esmaeili H. // Acta chim. Slov. 2019. Vol. 66. pp. 886-898.
Использование расчетных кинетических диаграмм для смешанной диффузии при обработке экспериментальных данных показало, что адсорбция сульфата на неполяризованном угле лучше описывается уравнением смешаннодиффузионной кинетики близкой к внешней диффузии, чем электросорбция этих ионов.

Емкость АУ по сульфату, достигаемая в процессе электросорбции, значительно превышает адсорбционную емкость без поляризации, кроме того, при электросорбции в условиях катодной поляризации существенная часть ионов $\mathrm{SO}_{4}{ }^{2-}$ сорбируется необратимо, избыточная часть адсорбтива не десорбируется при изменении поляризации. Исходя из этого факта, сделан вывод о влиянии химического механизма торможения на кинетику процесса электросорбции сульфата на АУ КМ-2 в условиях катодной поляризации.

Квантово-химическими расчетами показано, что ионы $\mathrm{SO}_{4}{ }^{2-}$ могут давать прочные соединения с углеродным кластером.

11. Salman M.S. // Al-Khwarizmi Eng. J. 2009. Vol. 5. pp. 72-76.

12. Mahmudov R., Huang Ch.P. // Sep.Pur.Technol. 2011. Vol. 77. pp. 294-300.

13. Ota K., Amano Y., Aikawa M. // Appl. Sur. Sci. 2013. Vol. 276. pp. 838-842.

14. Юсупова (Рамазанова) А.А., Свешникова Д.А., Рамазанов А.Ш. // Вестник ДГУ. Естественные науки. 2006. № 1. С.38-44.

15. Свешникова Д.А., Гафуров М.М., Шабанова 3.Э., Асваров А.Ш. и др. // Изв. выссших учебных заведений. Химия и химическая технология. 2009. Т. 52. С. 38-41.

16. Свешникова Д.А., Рамазанов А.Ш., Гафуров М.М., Кунжуева К.Г. и др. // Сорбицонные и хроматографические прочессы. 2012. Т. 12. № 5. С. 789-796.

17. Свешникова Д.А., Гафуров М.М., Атаев М.Б., Рабаданов К.Ш. и др. // Химия, физика и технология поверхности. 2013. Т. 4. № 1. C. 27-36.

18. Свешникова Д.А., Абакаров А.Н., Дрибинский А.В., Гафуров М.М. и др. // Журн. физ. химии. 1993. Т. 67. № 7. С. 1439-1443.

19. Резников А.А., Муликовская Е.П., Сщколов Ю. Методы анализа природных 
вод. М. Гос.научно-тех. изд-во литературы по геологии и охране недр. 1963. 404 с.

20. Rudzinski W., Plazinski W. // Appl. Surf. Sci. 2007. Vol. 253. pp. 5827-5840.

21. Rudzinski W., Plazinski W. // J. Phys. Chem. C. 2007. Vol. 111. pp. 15100-15110.

22. Plazinski W., Dziuba J., Rudzinski W. // Adsorption. 2013. Vol. 19. pp. 1055-1064.

23. Azizian S. // J. Colloid Interface Sci. 2004. Vol. 276. pp. 47-52.

24. Хамизов Р.Х., Свешникова Д.А., Кучерова А.Е., Синяева Л.А. // Журн. физ. химии. 2018. Т. 92. С. 1451-1460.

25. Хамизов Р.Х. // Журн. физ. химии. 2020. T. 94. C. 125-130.

26. Веницианов Е.В., Рубинштейн Р.Н. Динамика сорбции из жидких сред. М. Наука. 1983.237 c.

27. Никашина В.А. // Сорбичионыле и хроматографические прочессы. 2008. Т. 8. Номер С. 227-240
28. Lagergren S. // Kungliga Svenska Vetenkapsakademiens. Handlingar. 1898. Vol. 24. pp. 1-39.

29. Ho Y.S., McKay G. // Process. Biochem. 1999. Vol. 34. pp. 451-465.

30. Boehm H.P. Chemical Identification of Surface groups. In: Advances in catalysis and related subjects.1966. Vol. 16. pp. 179-274.

31. Runtti H., Tuomikoski S., Kangas T. et al. // BioResources. 2016. Vol. 11. pp. 3136-3152

32. Walter T. // WIREs Comput. Mol. Sci. 2014. Vol. 4. pp. 145-157.

33. Dral P.O., Wu X., Thiel W. // J. Chem. Theory Comput. 2019. Vol. 15. pp. 1743-1760.

34. Соловьев М.Е., Соловьев М.М. Компьютерная химия. М. СОЛОН-Пресс. 2005. 536 с.

35. Хамизов Р.Х., Свешникова Д.А., Кучерова А.Е., Синяева Л.А. // Журн. физ. химии. 2018. T. 92. C. 1619-1625.

\title{
The kinetics and mechanism of sulphate ion adsorption on KM-2 activated carbon
}

\author{
(C) 2021 Sveshnikova D.A. ${ }^{1,2}$, Suleimanov S.I. ${ }^{2,3}$, Khamizov R.Kh. ${ }^{4}$ \\ ${ }^{1}$ Institute of Geothermal and Renewable Energy Problems, Branch of the Joint Institute \\ for High Temperatures of the Russian Academy of Sciences, Makhachkala \\ ${ }^{2}$ Analytical Centre for Collective Use of the Dagestan Federal Research Centre \\ of the Russian Academy of Sciences, Makhachkala \\ ${ }^{3}$ Dagestan State University, Makhachkala \\ ${ }^{4}$ Vernadsky Institute of Analytical Chemistry and Geochemistry of the Russian Academy of Sciences, Moscow
}

The article is devoted to the study of the kinetics of adsorption and electrosorption (adsorption under cathodic potential) of $\mathrm{SO}_{4}{ }^{2-}$ ions on KM-2 activated carbon. The study was carried out in a batch mode. The experimental data regarding the kinetics of the $\mathrm{SO}_{4}{ }^{2-}$ adsorption on nonpolarised and polarised carbon were processed using kinetic diagrams for mixed diffusion and a linear isotherm. The comparison of the experimental data with theoretical dependences showed that the equation of mixed diffusion kinetics close to external diffusion better describes the adsorption of sulphate on a nonpolarised carbon than the electrosorption of these ions.

It was found that cathodic polarisation increases the sorption of these ions. In addition, during electrosorption under the conditions of cathodic polarisation, a significant part of $\mathrm{SO}_{4}{ }^{2-}$ ions are absorbed irreversibly and the excess part of the adsorptive is not desorbed when the polarisation changes. The studies showed that the adsorption processes of the $\mathrm{SO}_{4}{ }^{2-}$ ions on nonpolarised and cathode-polarised $\mathrm{KM}-2$ carbon can be satisfactorily described by models of formal kinetics which allows for the possibility to chemically limit the rate of such processes. Based on the fact that the adsorption of the studied ions was irreversible, it was concluded that the chemical mechanism of deceleration influences the kinetics of the process of sulphate electrosorption on KM-2 activated carbon under the conditions of cathodic polarisation.

A semiempirical PM3 method and the Orca 4.2 software were used for a quantum chemical study of the adsorption process of a sulphate ion on the surface of activated carbon simulated by an oxygen-containing carbon cluster $\mathrm{C}_{98} \mathrm{O}_{5} \mathrm{H}_{3}$. It was shown that $\mathrm{SO}_{4}{ }^{2-}$ ions are strongly adsorbed directly on the surface of activated carbon.

Keywords: adsorption, electrosorption, sulfate-ion, mixed diffusion kinetics, pseudo-first, pseudosecond order, quantum chemical calculations. 


\section{References}

1. Benatti C.T., Tavares C.R.G., Lenzi E., J. Environ. Manag., 2009, Vol. 90, pp. 504-511.

2. Tait S., Clarkeb W.P., Keller J., Batstonea D.J., Water Research, 2009, Vol. 43, pp. 762772.

3. Basha C.A., Selvi S.J.,Ramasamy E., Chellammal S., Chem. Eng. J., 2008, Vol. 141, pp. 89-98.

4. Lee H.J., Oh S.J., Moon S.H., Wat. Res., 2003, Vol. 37, pp. 1091-1099.

5. Galliana-Aleixandre M.V., Iborra-Clar A., Bes-Pifi A. et al., Desalination, 2005, Vol. 179, pp. 307-313.

6. Bodalo A., Gomez J.L. Gomez E., Leon G., Tejera M., Desalination, 2004, Vol. 162, pp. 5560.

7. Boukhalfa C., Desalination, 2010, Vol. 250, pp. 428-432.

8. Kolics A., Polkinghorne J.C., Wieckowski A., Electrochimica Acta, 1998, Vol. 43, pp. 2605-2618.

9. Ljng R., Liu Y., Wang X., Huang J., $J$. Chem. Eng. Data, 2011, Vol. 56, pp. 3890-3896.

10. Rahmati, Yeganeh G., Esmaeili H., Acta chim. Slov., 2019, Vol. 66, pp. 886-898.

11. Salman M.S., Al-Khwarizmi Eng. J., 2009, Vol. 5, pp. 72-76.

12. Mahmudov R., Huang Ch.P., Sep. Pur. Technol., 2011, Vol. 77, pp. 294-300.

13. Ota K., Amano Y., Aikawa M., Appl. Sur. Sci., 2013, Vol. 276, pp. 838-842.

14. Yusupova (Ramazanova) A.A., Sveshnikova D.A., Ramazanov A Sh., Vestnik DGU. Estestvenniye nauki, 2006, No 1, pp. 38-44.

15. Sveshnikova D.A., Gafurov M.M., Shabanova Z.E., Asvarov A.Sh. et al., Izv. vysshikh utshebnykh zavedenii. Khimiya and Khimitsheskaya tekhnologiya, 2009, Vol. 52, pp. 38-41.

16. Sveshnikova D.A., Ramazanov A.Sh., Gafurov M.M., Kunzhueva K.G. et al., Sorbtsionnye i khromatograficheskie protsessy, 2012, Vol. 12, No 5, pp. 789-796.

17. Sveshnikova D.A., Gafurov M.M., Ataev M.B. Rabadanov K.Sh. et al., Chemistry, Phyzik and Surface Technology, 2013, Vol. 4, No 1, pp. 27-36.
18. Sveshnikova D.A., Abakarov A.N., Dribinski A.V., Gafurov M.M. et al., Rus. J. Phys. Chem., 1993, Vol. 67, No 7, pp. 1439-1443.

19. Reznikov A.A., Mulikovskaya E.P., Sokolov Yu., Metody analiza prirodnykh vod, M., Gos.nauchno-tekh. izd-vo literatury po geologii i okhrane nedr, 1963, $404 \mathrm{~s}$.

20. Rudzinski W., Plazinski W., Appl. Surf. Sci., 2007, Vol. 253, pp. 5827-5840.

21. Rudzinski W., Plazinski W., J. Phys. Chem. C, 2007, Vol. 111, pp. 15100-15110.

22. Plazinski W., Dziuba J., Rudzinski W., Adsorption, 2013, Vol. 19, pp. 1055-1064.

23. Azizian S., J. Colloid Interface Sci, 2004, Vol. 276, pp. 47-52.

24. Khamizov R.Kh., Sveshnikova D.A.,Kutsherova A.E., Sinyaeva L.A., Rus. J. Phys. Chem.. 2018, Vol. 92, pp.1451-1460. DOI: $10.1134 / \mathrm{S} 0044453718090121$

25. Khamizov R.Kh., Rus. J. Phys. Chem., 2020, Vol. 94, pp. 125-130. DOI: $10.31857 / \mathrm{S} 0044453720010148$

26. Venitsianov E.V., Rubinshtein R.N., Dinamika sorbtsii iz zhidkikh sred, M., Nauka Publ, 1983, $237 \mathrm{p}$.

27. Nikashina V.A., Sorbtsionnye i khromatograficheskie protsessy, 2008, Vol. 8, pp. 227240.

28. Lagergren S., Kungliga Svenska Vetenkapsakademiens. Handlingar, 1898, Vol. 24, pp. 1-39.

29. Ho Y.S., McKay G., Process. Biochem., 1999, Vol. 34, pp. 451-465.

30. Boehm H.P. Chemical Identification of Surface groups. In: Advances in catalysis and related subjects. 1966, Vol. 16, pp.179-274.

31. Runtti H., Tuomikoski S., Kangas T. et al., BioResources, 2016, Vol. 11, pp. 3136-3152.

32. Walter T., WIREs Comput. Mol. Sci., 2014, Vol. 4, pp. 145-157.

33. Dral P.O., Wu X., Thiel W., J. Chem. Theory Comput., 2019, Vol. 15, pp. 1743-1760.

34.Solov'ev M.E., Solov'ev M.M., Komputernaya khimiya, M., SOLON-Press Publ, 2005, $536 \mathrm{p}$.

35. Khamizov R.Kh., Sveshnikova D.A., Kutsherova A.E., Sinyaeva L.A., Rus. J. Phys. Chem., 2018, Vol. 92, pp. 1619-1625. DOI: $10.1134 / \mathrm{S} 0044453718100114$ 
Свешникова Джаннет Алексеевна - к.х.н., ведущий научный сотрудник, Институт проблем геотермии и возобновляемой энергетики. Филиал ОИВТ РАН, Махачкала

Сулейманов Сагим Икрамович - к.Х.н., научный сотрудник, Аналитический центр коллективного пользования, ДФИЦ РАН, Махачкала

Хамизов Руслан Хажсетович - д.Х.н., заведующий лабораторией сорбционных методов, ГЕОХИ РАН, Москва.
Sveshnikova Dzhannet A. - Candidate of chem.. sci., Leading researcher, Institute of Geothermal and Renewable Energy Problems Branch of the JIHT RAS, Makhachkala, e-mail: jannet49@yandex.ru

Suleimanov Sagim I. - Candidate of chem. for Collective Use, DFRC RAS, Makhachkala

Khamizov Ruslan Kh. - Dr. Sci. (Chem) Head of Laboratory of Sorption Methods, GEOKHI RAS, Moscow 